JASC 12-2-6

\title{
Design of Hard Partition-based Non-Fuzzy Neural Networks
}

\author{
Keon-Jun Park*, Jae-Hyun Kwon *, Yong-Kab Kim ${ }^{\dagger}$
}

*Dept. of Information and Communication Engineering, Wonkwang University, Korea

${ }^{\dagger}$ Dept. of Information and Communication Engineering, Wonkwang University, Korea

\begin{abstract}
This paper propose a new design of fuzzy neural networks based on hard partition to generate the rules of the networks. For this we use hard c-means (HCM) clustering algorithm. The premise part of the rules of the proposed networks is realized with the aid of the hard partition of input space generated by HCM clustering algorithm. The consequence part of the rule is represented by polynomial functions. And the coefficients of the polynomial functions are learned by BP algorithm. The number of the hard partition of input space equals the number of clusters and the individual partitioned spaces indicate the rules of the networks. Due to these characteristics, we may alleviate the problem of the curse of dimensionality. The proposed networks are evaluated with the use of numerical experimentation.
\end{abstract}

Key words: Non-Fuzzy Neural Networks, Hard partition, HCM clustering, Scatter partition, Rule Generation

\section{INTRODUCTION}

Fuzzy neural networks (FNN) have emerged as one of the active areas of research related to integration of in fuzzy inference systems and neural networks [1, 2]. There have been many approaches to synthesize and apply for these fields $[3,4,5,6]$. Typically, FNNs are represented by fuzzy "if-then" rules while the back propagation (BP) is used to optimize the parameters.

The generation of the fuzzy rules and the adjustment of its membership functions were done by trial and error and/or operator's experience. The designers find it difficult to develop adequate fuzzy rules and membership functions to reflect the essence of the data. Moreover, some information gets lost or ignored on purpose when human operators articulate their experience in the form of linguistic rules. As a consequence, there is a need for an environment to construct and/or adjust a collection of linguistic rules.

In this paper, we propose the design of non-fuzzy neural networks by means of hard partition of input space using hard c-means (HCM) clustering algorithm [7]. The premise part of

Manuscript received : Sept. 3, 2012 / revised : Nov. 1, 2012

†Corresponding Author: ykim@wonkwang.ac.kr

Tel: +82-063-850-6695, Fax: +82-063-850-6074, Wonkwang Univ.

*Dept. of Information and Communication Eng., Wonkwang Univ., Korea the rules is realized with the aid of the hard scatter partition of input space generated by HCM clustering algorithm. The consequence part of the rule is represented by polynomial functions. And the coefficients of the polynomial functions are learned by BP algorithm. The proposed network is evaluated through the numeric experimentation.

The paper is organized as follows. Section 2 is concerned with the design of non-fuzzy neural networks based on hard partition. Section 3 presents results of numeric experimentations. Finally Section 4 concludes the paper.

\section{NON-FUZZY NEURAL NETWORKS}

In this section, the design of non-fuzzy clustering-based if-then rules along with their development mechanism is discussed. More specifically, we elaborate on the four types of fuzzy inference and present the learning algorithm.

\section{A. Hard c-means clustering algorithm}

$\mathrm{HCM}$ algorithm [7] is aimed at the formation of ' $c$ ' clusters (relations) in $\mathbf{R}^{n}$. We obtain the matrix representation for hard $c$-partition, defined as follows.

$$
M_{C}=\left\{\mathbf{U} \mid u_{i k} \in\{0,1\}, \sum_{i=1}^{c} u_{i k}=1, \quad 0<\sum_{k=1}^{n} u_{i k}<n\right\}
$$


[Step 1] Fix the number of clusters $c(2 \leq c<n)$ and initialize the partition matrix $\mathbf{U}^{(0)} \in M_{C}$

[Step 2] Calculate the center vectors vi of each cluster:

$$
\begin{aligned}
& \mathbf{v}_{i}^{(r)}=\left\{v_{i 1}, v_{i 2}, \cdots, v_{i j}, \cdots, v_{i m}\right\} \cdot \\
& v_{i j}^{(r)}=\sum_{k=1}^{n} u_{i k}^{(r)} \cdot x_{k j} / \sum_{k=1}^{n} u_{i k}^{(r)} .
\end{aligned}
$$

Where, $\left[u_{i k}\right]=\mathbf{U}^{(r)}, i=1,2, \ldots, c, j=1,2, \ldots, m$.

[Step 3] Update the partition matrix $\mathbf{U}^{(r)}$; these modifications are based on the standard Euclidean distance function between the data points and the prototypes,

$$
\begin{aligned}
& d_{i k}=d\left(\mathbf{x}_{k}-\mathbf{v}_{i}\right)=\left\|\mathbf{x}_{k}-\mathbf{v}_{i}\right\|=\left[\sum_{j=1}^{m}\left(x_{k j}-v_{i j}\right)^{2}\right]^{1 / 2} \\
& u_{i k}^{(r+1)}=\left\{\begin{array}{lc}
1 & d_{i k}^{(r)}=\min \left\{d_{j k}^{(r)}\right\} \text { for all } j \in c \\
0 & \text { otherwise }
\end{array}\right.
\end{aligned}
$$

[Step 4] Check a termination criterion. If

$$
\left\|\mathbf{U}^{(r+1)}-\mathbf{U}^{(r)}\right\| \leq \varepsilon(\text { tolerance level })
$$

\section{B. The structure of the HCM-based NFNN}

The structure of the HCM-based NFNN involves HCM clustering algorithm in the premise part and neural networks present in the consequence part of the rules. The overall topology of the network is illustrated in Fig. 1.

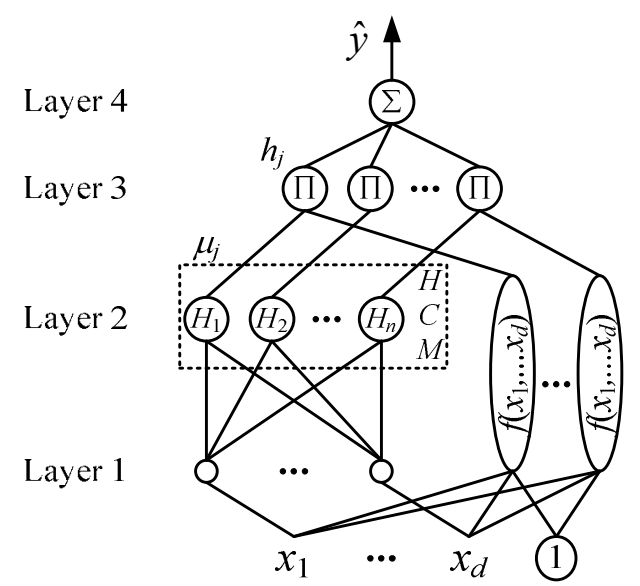

Fig. 1. The structure of HCM-based NFNN.

The proposed HCM-based NFNN is implied by the hard scatter partition of input spaces. In this sense, each rule can be viewed as a certain rule of the following format.

$$
R^{j}: \text { If } x_{1} \text { and } \cdots \text { and } x_{d} \text { is } H_{j} \text { Then } y_{j}=f\left(x_{1}, \ldots, x_{d}\right)
$$

As far as inference schemes are concerned, we distinguish these cases :

Type 1 (Simplified Inference): $f=w_{j 0}$

Type 2 (Linear Inference): $f=w_{j 0}+\sum_{k=1}^{d} w_{j k} x_{k}$

Type 3 (Quadratic Inference):

$f=w_{j 0}+\sum_{k=1}^{d} w_{j k} x_{k}+\sum_{k=1}^{d} w_{j,(d+k)} x_{k}^{2}+\sum_{k=1}^{d} \sum_{l=k+1}^{d} w_{j z} x_{k} x_{l}$

Type 4 (Modified Quadratic Inference):

$f=w_{j 0}+\sum_{k=1}^{d} w_{j k} x_{k}+\sum_{k=1}^{d} \sum_{i=k+1}^{d} w_{j z} x_{k} x_{i}$.

To be more specific, $R^{j}$ is the $j$-th fuzzy rule, while $H_{j}$ denotes $j$-th membership grades using HCM clustering algorithm., w's are consequent parameters of the rule. The functionality of each layer is described as follows. [Layer 1] The nodes in this layer transfer the inputs. [Layer 2] The nodes here are used to calculate the membership degrees using HCM clustering algorithm.

[Layer 3] The nodes in this layer realize a certain inference process.

$h_{j}=\mu_{j} y_{j}$

[Layer 4] The nodes in this layer compute the outputs.

$\hat{y}=\sum_{j=1}^{n} h_{j}$

\section{The learning algorithm}

The parametric learning of the network is realized by adjusting connections of the neurons and as such it could be realized by running a standard back-propagation (BP) algorithm. The performance index is based on the Euclidean distance. As far as learning is concerned, the connections are adjusted in a standard fashion,

$w_{j 0}(p+1)=w_{j 0}(p)+\Delta w_{j 0}$

where this update formula follows the gradient descent method.

Quite commonly to accelerate convergence, a momentum coefficient $\alpha$ is being added to the learning expression.

$$
\begin{aligned}
& \Delta w_{j 0}=\eta\left(y_{p}-\hat{y}_{p}\right) \hat{\mu}_{j}+\alpha\left(w_{j 0}(p)-w_{j 0}(p-1)\right) \\
& \Delta w_{j k}=\eta\left(y_{p}-\hat{y}_{p}\right) \hat{\mu}_{j} x_{k}+\alpha\left(w_{j k}(p)-w_{j k}(p-1)\right) \\
& \Delta w_{j,(d+k)}=\eta\left(y_{p}-\hat{y}_{p}\right) \hat{\mu}_{j} x_{k}^{2}+\alpha\left(w_{j,(d+k)}(p)-w_{j,(d+k)}(p-1)\right) \\
& \Delta w_{j z}=\eta\left(y_{p}-\hat{y}_{p}\right) \hat{\mu}_{j} x_{k} x_{i}+\alpha\left(w_{j z}(p)-w_{j z}(p-1)\right)
\end{aligned}
$$

\section{Experimental Studies}

We discuss numerical example in order to evaluate the 
advantages and the effectiveness of the proposed approach. This time series data (296 input-output pairs) coming from the gas furnace nonlinear process has been intensively studied [8]. The delayed terms of methane gas flow rate $u(t)$ and carbon dioxide density $y(t)$ are used as input variables organized in a vector format as $[u(t-3), u(t-2), u(t-1), y(t-3)$, $y(t-2), y(t-1)] . y(t)$ is the output variable. The first part of the data set (consisting of 148 pairs) was used for training purposes. The remaining part of the series serves as a testing data set. We consider the MSE as a performance index.

we construct the network for a two and six-dimensional system from the dataset, respectively.

Fig 2 and Fig 3 summarize the performance index for training and testing data by setting the number of clusters and inference type. Here, 2, 3, 5 and 10 are the number of the rules, PI and E_PI stand for the performance index for the training data set and the testing data set, respectively.

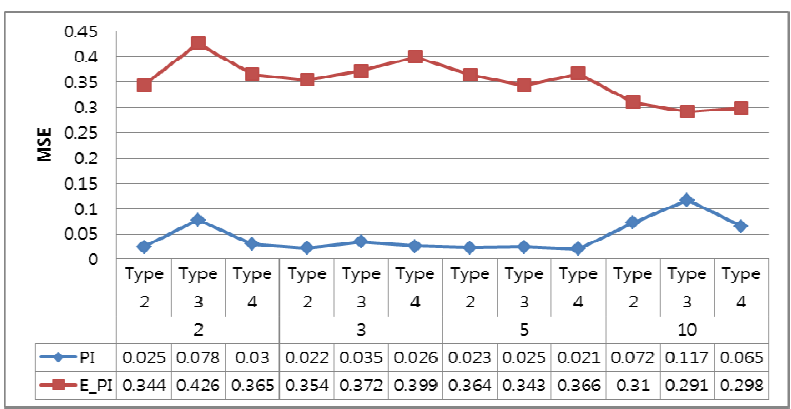

Fig. 2. Performance index for two-dimension system.

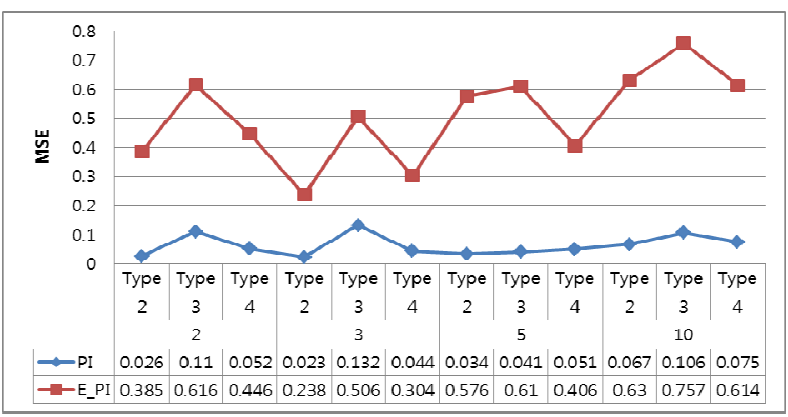

Fig. 3. Performance index for six-dimension system.

From the Fig 2, we selected the best network with ten rules (clusters) with modified quadratic inference that exhibits PI $=$ 0.117 and E_PI $=0.291$ for two-dimension system.

From the Fig 3, we selected the best network with three rules (clusters) with linear inference that exhibits $\mathrm{PI}=0.023$ and E_PI $=0.238$ for two-dimension system.

Fig. 4 shows 10 hard-partitioned input spaces using HCM clustering algorithm in the selected model in two-dimensional input space. The boundaries of each local space indicate the binary boundary by the membership matrix with a value of 0 or 1 .

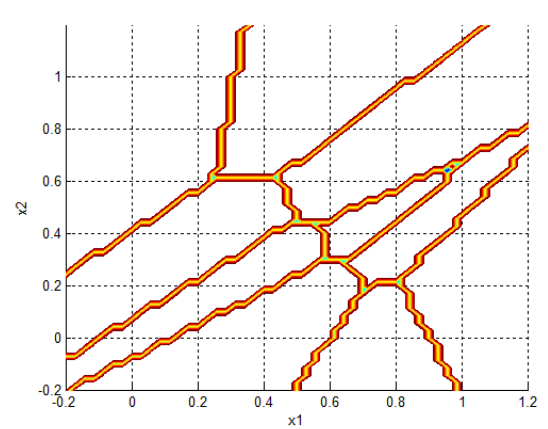

Fig. 4. Partitioned input spaces by means of HCM clustering algorithm for two-dimension system.

Fig. 5 and Fig. 6 depict the original and model outputs of training and testing data for the selected model. This figure shows that the model output is approximately the same for original output.

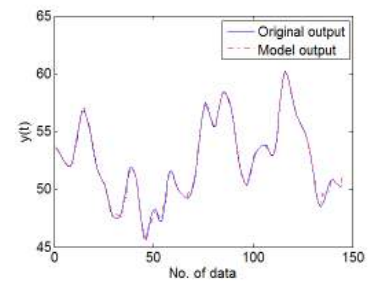

(a) Training data

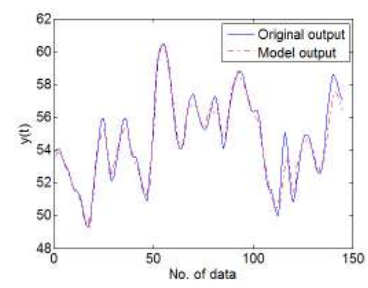

(b) Testing data
Fig. 5. Model output (10 clusters, modified quadratic inference) for two-dimension system.

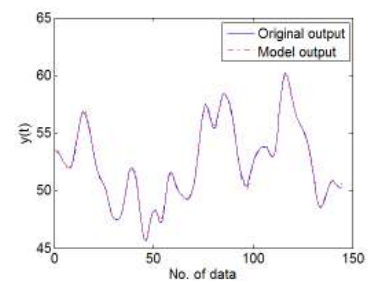

(a) Training data

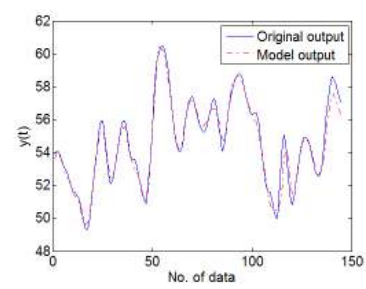

(b) Testing data
Fig. 6. Model output (3 clusters, linear inference) for six-dimension system.

\section{Conclusion}

This paper have designed a non-fuzzy neural networks based on hard-partition of input space to generate the rules of the system for nonlinear process. The input spaces of the proposed networks were divided as the hard scatter form using HCM clustering algorithm By this method, we could alleviate the problem of the curse of dimensionality and design the networks that are compact and simple.

From the results in the previous section, we were able to design preferred network. Through the use of a performance 
index, we were able to achieve a balance between the approximation and generalization abilities of the resulting model. Finally, this approach would find potential application in many fields.

\section{REFERENCES}

[1] T. Yamakawa, "A Neo Fuzzy Neuron and Its Application to System Identification and Prediction of the System Behavior," Proceeding of the 2nd International Conference on Fuzzy logic \& Neural Networks, pp. 17-22, 1992.

[2] J. J. Buckley and Y. Hayashi, "Fuzzy neural networks: A survey," Fuzzy Sets Syst. Vol. 66, 1994.

[3] J.S.R. Jang, E. Mizutani, and C.T. Sun, Neuro-Fuzzy and Soft Computing, A Computational Approach to Learning and Machine Intelligence, Prentice Hall, NJ, 1997.

[4] J. J. Park, G. S. Choi, and I.-S. Ahn, "Multi-variable Fuzzy Modeling for Combustion Control of Refuse Incineration Plant," Journal of the Institute of Webcasting, Internet and Telecommunication(IWIT), Vol. 9, No. 5, pp. 191-197, 2009.

[5] T. A. Tuan and I. S. Koo, "A Channel Selection Algorithm Based on Fuzzy Logic and Learning Automata for Cognitive Radio Sensor Networks", Journal of the Institute of Webcasting, Internet and Telecommunication(IWIT), Vol. 11. No. 1, pp. 23-28, 2011.

[6] I. K. Park, " A Study on the Prediction of the Nonlinear Chaotic Time Series Using Genetic Algorithm based Fuzzy Neural Network", Journal of the Institute of Webcasting, Internet and Telecommunication(IWIT), Vol. 11. No. 4, pp. 91-97, 2011.

[7] P. R. Krishnaiah and L. N. Kanal, editors. Classification, pattern recognition, and reduction of dimensionality, volume 2 of Handbook of Statistics. North-Holland, Amsterdam, 1982.

[8] G. E. P. Box and G. M. Jenkins, Time Series Analysis, Forecasting, and Control, 2nd edition Holden-Day, SanFransisco, 1976.

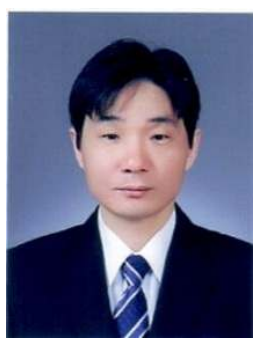

Keon-Jun Park received the B.S degree in Electrical \& Electronics Engineering from the Wonkwang University, Iksan, South Korea, in 2003, and the M.S degree in Control and Instrumentation Engineering in 2005. He received the $\mathrm{Ph} . \mathrm{D}$ degree in Electrical Engineering from University of Suwon, Suwon, South Korea, in 2010 . He is currently a research professor with Information and Communication Engineering, Wonkwang University, Korea. His research interests include computational intelligence, intelligence modeling and control, and pattern recognition.

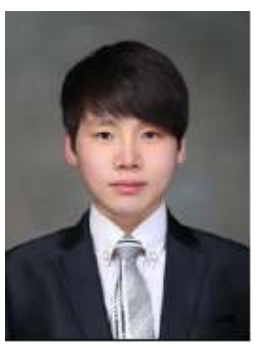

Jae-Hyun Kwon received the B.S degree in Electrical Information Communication Engineering from WonKwang University. $\mathrm{He}$ is currently the master's degree in Information and Communication Engineering, WonKwang University, Korea. His research interests are Medical LED, Optical Fiber Sensing, Visible lighting Communication.

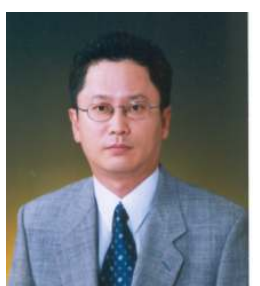

Yong-K. Kim received the B.S degree in Electronics Engineering from Ajou University and the M.S.E degree in Electrical \& Computer Engineering from University of Alabama in Huntsville. He received his Ph.D degree in Electrical \& Computer Engineering in North Carolina State University. $\mathrm{He}$ is currently in Professor at School of Information and Communication Engineering, Wonkwang University, Korea. His research interests are Remote Sensing for Visible Communication, Optical Fiber Sensing, Intelligent Control, Advanced Computational Intelligence. 